\title{
Lipid-laden macrophage index and inflammation in bronchoalveolar lavage fluids in children
}

\author{
M.Y. Kazachkov*, M.S. Muhlebach*, C.A. Livasy\#, T.L. Noah*
}

Lipid-laden macrophage index and inflammation in bronchoalveolar lavage fluids in children. M. Y. Kazachkov, M.S. Muhlebach, C.A. Livasy, T.L. Noah. (C) ERS Journals Ltd 2001.

ABSTRACT: The presence of lipids in alveolar macrophages has been used clinically as an indicator of aspiration, a process associated with increased lung inflammation in animal models. The hypothesis is that the quantity of lipids in alveolar macrophages, measured as lipid-laden index (LLI), would correlate with lung inflammation in paediatric patients.

Children with chronic respiratory symptoms (21 cystic fibrosis (CF), 24 non-CF) underwent flexible bronchoscopy with bronchoalveolar lavage (BAL) and 24-h intraoesophageal $\mathrm{pH}$ monitoring for clinical indications. Total cell counts, number and per cent of neutrophils and macrophages, and LLI were determined in the bronchoalveolar lavage fluids (BALF) from all children. BALF were also obtained from eight healthy, young nonsmoking adults for comparison.

LLI in non-CF children were $6.9 \pm 3.5$ (mean \pm SEM) which were higher than LLI in healthy adults $(1.0 \pm 0.4),(p=0.045)$. Children with $C F$ had very high LLIs $(19.2 \pm 4.5)$ compared with both healthy adults $(p=0.014)$ and children without $C F(p=0.045)$. LLI did not correlate with airway inflammation in any group. LLI in children with abnormal $\mathrm{pH}$ probes had a tendency to be higher than in children with normal $\mathrm{pH}$ probes, but the difference was not significant $(\mathrm{p}=\mathbf{0 . 0 9 8}$ ).

It is concluded that the lipid-laden index was significantly elevated in children with chronic respiratory symptoms compared with healthy adults, and in children with cystic fibrosis compared with those who have other chronic respiratory conditions. However, the lipid-laden index did not correlate with the quantity of bronchoalveolar lavage fluid inflammation. The lipid-laden index in children may, in part, reflect processes other than aspiration, such as airways obstruction.

Eur Respir J 2001; 18: 790-795.
Depts of *Pediatrics and "Pathology, University of North Carolina at Chapel Hill, USA.

Correspondence: M.Y. Kazachkov, Dept of Pediatrics, University of North Carolina at Chapel Hill, 635 Burnett Womack Building, CB \#7220, Chapel Hill, NC 27599-7220, USA.

Fax: 19199666179

Keywords: Airway inflammation bronchoalveolar lavage

children

lipid-laden macrophages

Received: May 252000

Accepted after revision June 202001

M.Y. Kazachkov was supported by NIH grant no. 5-53970-XXXX-0-401 4228. This work was also supported in part by the University of North Carolina Center for Environmental Medicine and Lung Biology.
Gastro-oesophageal reflux and aspiration may contribute to the development of chronic respiratory diseases, such as asthma, aspiration pneumonitis, chronic bronchitis, bronchiectasis, and a variety of otolaryngological disorders in paediatric patients [1-4]. Accumulation of lipids in the cytoplasm of alveolar macrophages is considered to be evidence of aspiration $[5,6]$. The amount of lipids in alveolar macrophages can be quantitated and expressed as the lipid-laden index (LLI) [7, 8]. This index takes into account both the number of macrophages containing lipids, and the relative amount of lipids present in the macrophages.

In animal studies, experimental aspiration of gastric acid and food particles causes intensive inflammation in airways with accumulation of inflammatory cells in the airway lumen and submucosal layer [9, 10]. The degree of tissue response is related to the $\mathrm{pH}$ and volume of the injected acid solution [9]; aspiration of gastric acid with particles of food enhances the inflammatory response [11].

The hypothesis of the present study was that the LLI would correlate with lung inflammation in children. In order to test this hypothesis, inflammation was compared with the LLI in the bronchoalveolar lavage fluids (BALF) of paediatric patients with chronic respiratory symptoms.

\section{Materials and methods}

\section{Patients}

Children with respiratory problems, who underwent clinically indicated flexible bronchoscopy and intraoesophageal pH monitoring between January 1995May 1999, were eligible for participation in this study. Gastro-oesophageal reflux (GER) and/or aspiration were thus suspected clinically as one of the possible causes of respiratory symptoms in all children included in this study. Patients with tuberculosis, acute illnesses, or systemic or inhaled steroid medication use within one month prior to bronchoscopy were not included. Because of the known correlation between airway infection and inflammation [12], all infected patients (defined as $>5 \times 10^{4}$ colony forming 
units $(\mathrm{cfu}) \cdot \mathrm{mL}^{-1}$ of pathogenic bacteria or positive viral culture in BALF) were excluded from analysis. In addition, eight young, nonsmoking, symptom-free adults underwent flexible bronchoscopy with bronchoalveolar lavage (BAL) and served as a healthy control group for comparison with paediatric patients.

The study was approved by the committee for the protection of the rights of the human subjects at the University of North Carolina (UNC) School of Medicine.

\section{Bronchoscopy and bronchoalveolar lavage}

Bronchoscopy and BAL in children were performed according to standard clinical protocol at UNC Hospitals. The location of BAL was at the discretion of the bronchoscopist, but was generally performed in the lung segment most affected by disease, as evidenced by radiographical changes or by visual appearance at bronchoscopy. The bronchoscope was wedged into a bronchus and two to three $10-\mathrm{mL}$ aliquots of buffered normal saline solution were instilled and immediately aspirated through the bronchoscope. In some smaller infants, $5-\mathrm{mL}$ aliquots were used. The total volume of instilled lavage fluid was $1-3 \mathrm{~mL} \cdot \mathrm{kg}^{-1}$ body weight. An aliquot of the BAL for the present study was taken in a sterile manner. The remainder of the BALF was sent to the hospital laboratory for cytology and cultures, which were ordered by the physician responsible for the patient.

Healthy, nonsmoking adult volunteers underwent fibreoptic bronchoscopy with lavage. None of the subjects had a history of asthma, allergic rhinitis, chronic respiratory disease, or cardiac disease. Subjects were excluded from the study if they had recently had an acute respiratory illness, and were asked to avoid exposure to air pollutants such as tobacco smoke and paint fumes. The fibreoptic bronchoscope was wedged into a segmental bronchus of the lingula. Six aliquots of sterile saline were instilled and immediately aspirated. The first was $20 \mathrm{~mL}$ and this fraction was labelled the bronchial sample. The remaining five aliquots were $50 \mathrm{~mL}$ each and were labelled the alveolar sample. These were put on ice immediately, with determination of total cell counts, differentials, and LLI taking place later.

\section{Lipid index scoring}

The BALF was transferred to $25 \mathrm{~mL}$ tubes and centrifuged at $1,200 \times g$ for $15 \mathrm{~min}$. The supernatant was discarded and the cells were concentrated into 3-4 drops of fluid. One to two drops of the concentrated cellular suspension was transferred to a centrifuge tube with a slide, and spun at $200 \times g$ for $10 \mathrm{~min}$. The cytocentrifuged preparations were then stained for lipids with Sudan 4 (Acros Organics, New Jersey, USA).

Each slide was evaluated for the number of lipidladen macrophages per 100 consecutive macrophages. A semiquantitative method of LLI calculation was used, which was analogous to that reported by
Colombo and Hallberg [8]. Preliminary studies suggested that dividing the percentage of lipid opacification of the macrophage into $<50 \%$ and $>50 \%$, rather than into quartiles as reported [8], resulted in less interobserver variability. For the calculation of LLI, macrophages with no visible lipid staining of the cytoplasm were scored "0". Macrophages with $<50 \%$ of the cytoplasm opacified by lipid were scored "1+". Macrophages with $>50 \%$ of the cytoplasm opacified by lipid were scored "2+". The LLI was then calculated as follows: $L L I=((\% 1+$ macrophages $) \times 1)+$

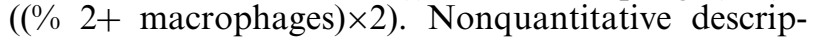
tors of lipid droplet size or appearance were not attended. The pathologist who calculated the LLI was not aware of clinical findings or the results of the BAL cultures and cell differentials of the patients.

\section{Microbiology}

BALF cultures were carried out according to standard protocol at the UNC Hospital's clinical microbiology laboratory [12]. Briefly, BALF specimens were cultured for Gram-positive and Gramnegative bacteria. In addition, cultures and stains for viruses (including cytomegalovirus, respiratory syncytial virus, influenza A and B, parainfluenza 1-3, and adenovirus), fungi, and mycobacteria were carried out when these infections were clinically suspected. Oropharyngeal flora (OPF) in BALF specimens were defined according to standard protocol for the UNC Hospital's Clinical Microbiology Laboratory. These consist of a mixture of resident flora, including Streptococcus viridans, nonhaemolytic streptococci, saprophytic Neisseria spp., diphtheroids, coagulasenegative Staphylococcus spp., micrococci, lactobacilli, haemolytic Haemophilis spp., H. parainfluenzae, Eikenella corrodens, non-Group A beta-haemolytic Streptococcus spp., Bacillus spp., and Stomatococcus spp. Quantitative bacterial cultures were performed on both pathogenic flora and OPF. OPF were not included in the total bacterial $\mathrm{cfu} \cdot \mathrm{mL}^{-1}$ BALF used to define whether subjects had infection.

\section{Bronchoalveolar lavage fluid cell count}

The samples were processed immediately in the bronchoscopy laboratory. BALF was gently mixed and the total number of white cells in the native, unfiltered lavage fluid was determined in a haemocytometer. Microscope slides were prepared in a Wescor Cytopro 7620 cytocentrifuge (Logan, UT, USA) by centrifuging $150 \mu \mathrm{L}$ fluid at $60 \times g$ for $4 \mathrm{~min}$. To obtain a cell count of $\sim 5 \times 10^{4}$ cells per slide, some samples had to be further diluted in normal saline lavage fluid before loading onto the cytocentrifuge. Slides were fixed and stained with modified Wright-Giemsa stain (Leukostat, Fisher Diagnostic, Pittsburgh, PA, USA). Two hundred consecutive cells were counted under oil immersion and both the number and per cent of macrophages, neutrophils, epithelial cells, lymphocytes and eosinophils were recorded. Differential cell 
counts were made without knowledge of the BALF culture results.

\section{Intra-oesophageal pH probe monitoring}

Intra-oesophageal $\mathrm{pH}$ probe monitoring was also performed according to standard clinical protocol at UNC Hospitals. The oesophageal $\mathrm{pH}$ electrode (Synectic Medical Irving, TX, USA) was placed $\sim 5 \mathrm{~cm}$ above the lower oesophageal sphincter for 18-24 h. Total acid exposure time and percentage of time at $\mathrm{pH}$ $<4.0$ (reflux index or $\mathrm{pH}$ index) was recorded. Reflux index $>5$ was considered to be abnormal for all age groups, in accordance with previous reports [13].

\section{Statistical methods}

Data are reported as mean \pm SEM unless otherwise stated. Linear regressions were calculated by the method of least squares, and slopes and elevations of the lines were compared using unpaired t-tests. The data were analysed using the Mann-Whitney U-test. Statistical significance was defined as $\mathrm{p}<0.05$ throughout.

\section{Results}

\section{Lipid-laden index in different groups}

BALF from 24 uninfected children without cystic fibrosis (CF), 21 uninfected children with $\mathrm{CF}$, and eight healthy adults were available. Clinical indications for bronchoscopy and bronchoscopic diagnoses are summarized in table 1 . LLI in non-CF children was $6.9 \pm 3.5$ (mean \pm SEM). This was higher than in healthy adults $(1.0 \pm 0.4)(\mathrm{p}=0.045)$. Children with $\mathrm{CF}$ had a high LLI (19.2 \pm 4.5$)$ compared with both healthy adults $(\mathrm{p}=0.014)$ and children without $\mathrm{CF}$ $(\mathrm{p}=0.045)$ (fig. 1). However, there was no difference in $\mathrm{pH}$ index between children with and without $\mathrm{CF}$.

There were two neurologically impaired non-CF children who had a high likelihood of aspiration, manifested by choking and coughing with feeds, and positive results for laryngeal/tracheal penetration in a

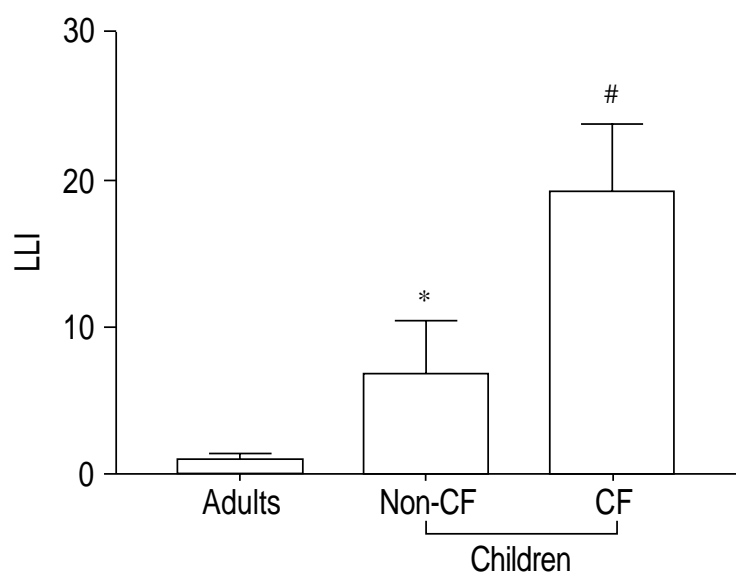

Fig. 1.-Lipid-laden index (LLI) for children with and without cystic fibrosis (CF and non-CF), and healthy adult controls. Each bar represents mean \pm SEM. *: $\mathrm{p}<0.05$ versus healthy adults; \#: $\mathrm{p}<0.05$ versus healthy adults and non-CF children.

barium-swallowing study. Their LLI, as well as their total cell counts, number and per cent of neutrophils, and number and per cent of macrophages, were not higher than those in other children without $\mathrm{CF}$.

\section{Lipid-laden index and airway inflammation}

As expected, based on previous studies [12], children with $\mathrm{CF}$ had higher total cell counts and numbers and per cent of neutrophils in their BALF than children without $\mathrm{CF}$. In both $\mathrm{CF}$ and non-CF patients, LLI did not correlate with the total cells or number or per cent of neutrophils in BALF. Similarly, there was no correlation between LLI and number or per cent of macrophages and epithelial cells in both groups.

All healthy adult controls had an LLI $\leqslant 3$. Therefore, the children without CF were divided into two groups with regard to their LLI. The first group had normal LLI of $\leqslant 3(n=16)$ and the second group had high LLI of $>3 \quad(n=8)$. There was no significant difference in the total number of cells, or number or per cent of neutrophils (figs. 2 and 3) in BALF between these two groups. Furthermore, the number

Table 1.-Ages, indications for bronchoscopy, and bronchoscopic diagnoses

\begin{tabular}{|c|c|c|c|c|c|c|}
\hline \multirow[t]{2}{*}{ Group } & \multirow{2}{*}{$\underset{n}{\text { Subjects }}$} & \multirow[t]{2}{*}{ Age } & \multicolumn{2}{|l|}{ Indication for bronchoscopy } & \multicolumn{2}{|c|}{ Diagnosis after bronchoscopy } \\
\hline & & & Indication & $\mathrm{n}$ & Diagnosis & $\mathrm{n}$ \\
\hline \multirow[t]{7}{*}{ Non-CF } & 24 & $28 \pm 7.35$ & Stridor & 7 & Bronchitis & 7 \\
\hline & & (2-94) months & Recurrent pneumonia & 6 & Laryngeal oedema & 4 \\
\hline & & & Persistent wheezing & 5 & Laryngomalacia & 4 \\
\hline & & & ALTE & 3 & Normal & 4 \\
\hline & & & Chronic cough & 3 & Glossoptosis & 2 \\
\hline & & & & & Vocal cord paresis & 2 \\
\hline & & & & & Bronchial compression & 1 \\
\hline $\mathrm{CF}$ & 21 & $\begin{array}{c}41 \pm 9.48 \\
(1-143) \text { months }\end{array}$ & $\begin{array}{l}\text { Exacerbation of lung disease, } \\
\text { inability to produce sputum }\end{array}$ & & Bronchitis & 21 \\
\hline Adult & 8 & $26 \pm 4$ yrs & Research study & & Healthy volunteers & \\
\hline
\end{tabular}

Age data are presented as mean \pm SEM (range). CF: cystic fibrosis; ALTE: apparent life-threatening event. 


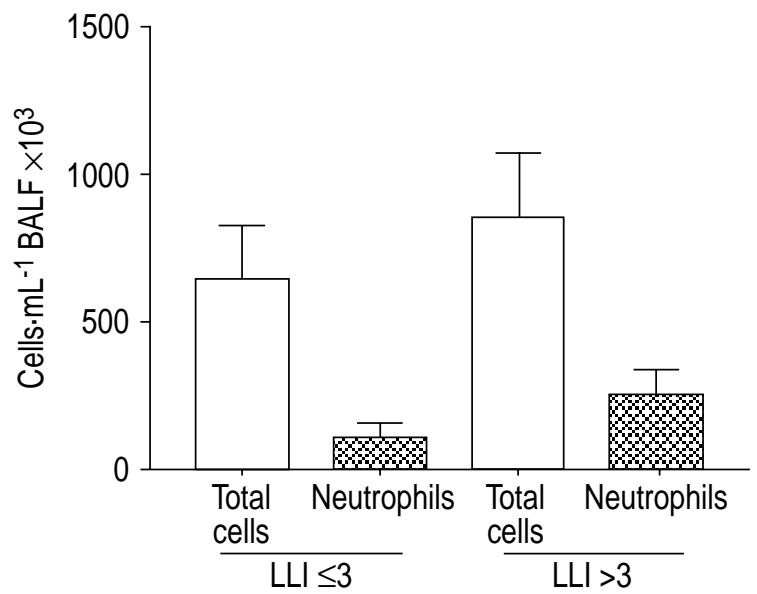

Fig. 2. - Total cells and neutrophils for children without cystic fibrosis divided in two groups (lipid-laden indices (LLI) $\leqslant 3$ versus LLI $>3$ ). Each bar represents mean \pm SEM. There is no significant difference between cell counts in two groups. BALF: bronchoalveolar lavage fluid.

and per cent of macrophages and epithelial cells were not different between the two groups. The same analysis was performed for the group of children with $\mathrm{CF}$ and, again, there was no difference in total cells, neutrophils, macrophages, or epithelial cells in BALF between groups of children with an LLI $\leqslant 3(n=7)$ and an LLI $>3(n=14)$. There was no difference in LLI between non-CF children with bronchitis (as reported by bronchologist, $n=7$ ) and other non-CF patients. In addition, total cells counts and the number and per cent of neutrophils and macrophages were not different.

\section{Other correlations}

The association between $\mathrm{pH}$ measurements and LLI was examined. The children without $\mathrm{CF}$ were divided into two groups. The first group consisted of patients with a normal reflux index of $\leqslant 5(n=12)$ and the

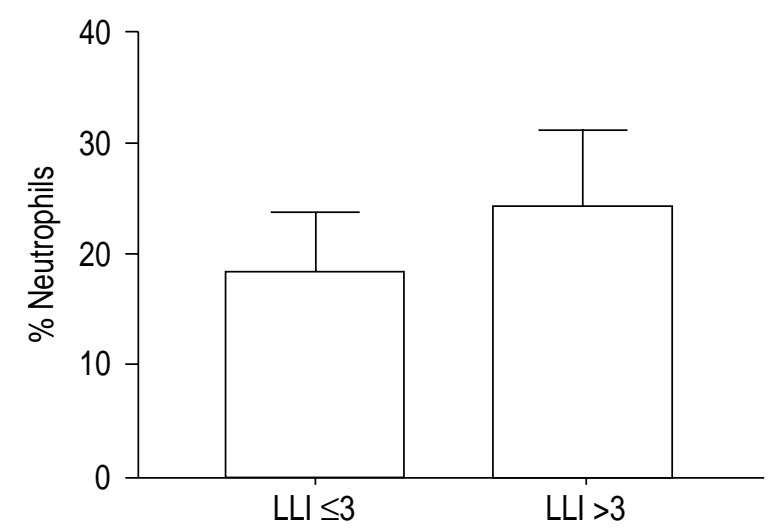

Fig. 3.-Per cent of neutrophils in bronchoalveolar lavage fluid for children without cystic fibrosis divided into the two groups (lipidladen indices $(\mathrm{LLI}) \leqslant 3$ versus LLI $>3$ ). Each bar represents mean \pm SEM. There is no significant difference in per cent of neutrophils in two groups.

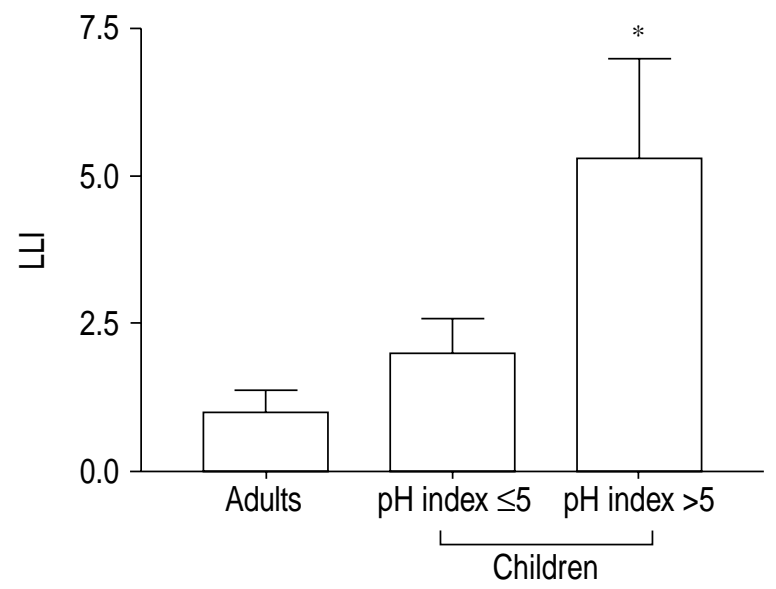

Fig. 4.-Lipid-laden indices (LLI) for children without cystic fibrosis with $\mathrm{pH}$ index $>5, \mathrm{pH}$ index $\leqslant 5$ and healthy adults. Each bar represents mean \pm SEM. *: $\mathrm{p}<0.05$ versus healthy adults, and $\mathrm{p}=0.098$ versus children with $\mathrm{pH}$ index $<5$.

second group of children with an abnormal reflux index of $>5(n=12)$. The LLI in children from the abnormal reflux group was higher, on average, than the LLI in children from the normal reflux group, but the difference was not statistically significant $(\mathrm{p}=$ 0.098) (fig. 4). However, the LLI in the children in the abnormal reflux group were higher than the LLI of healthy adults $(\mathrm{p}<0.05)$, suggesting GER had some effect on LLI. No significant difference in LLI was found between children with normal reflux and healthy adults. There was no statistically significant correlation between reflux index and either total cells, neutrophils, macrophages or epithelial cells, in children with or without $\mathrm{CF}$.

The amounts of OPF in BALF from children with both normal and abnormal reflux were not significantly different, either in the $\mathrm{CF}$ or non-CF group. Finally, there was no significant correlation between LLI and amount of OPF in any group.

\section{Discussion}

This study has found that the LLI in BALF macrophages is elevated in children with chronic respiratory symptoms undergoing clinically indicated bronchoscopy, compared to healthy adult volunteers. However, little relationship was observed between LLI and inflammation in BALF. Animal studies have shown that aspiration of gastric contents containing food particles leads to development of an acute inflammatory response in the airways, mediated by neutrophils [9, 11, 14-16]. The severity of airway inflammation correlated with volume and $\mathrm{pH}$ of gastric contents in one of these reports [9]. Deepithelialization of bronchial mucosa has also been shown to occur [17]. In rabbits, IsHII et al. [10] showed that the number and per cent of neutrophils in BALF increased during the first day after aspiration of acid, and subsequently decreased over the next 6 days, while the number and per cent of macrophages were increasing. 
The method of calculating LLI used in this study was analogous, but not identical, to that described by Colombo and Hallberg [8]. This method was devised to increase confidence in the accuracy of the data; it is often difficult to differentiate the percentage of lipid opacification of macrophage cytoplasm into quartiles. Since a primary goal of this study was the correlation of LLI with quantitative measures of inflammation in BALF, accurate quantification of LLI was essential. However, using a different LLI scoring method makes direct comparison of these data with other studies difficult.

For ethical reasons, the use of healthy children as a control group for LLI was not permitted. Thus, it is conceivable that comparison with adult controls affected the conclusion that LLI are generally elevated in children with chronic respiratory symptoms, since the LLI in healthy children was not determined.

The LLI in children with abnormal reflux was significantly higher than the LLI in healthy adults, and had a tendency to be higher than the LLI in children with normal reflux, although the latter was not statistically significant $(\mathrm{p}=0.098)$. This suggests that GER and aspiration may contribute to the accumulation of lipids in alveolar macrophages. This is also consistent with the conclusion of COLOMBO and HALlBerg [18], who found only modest and not statistically significant elevation of LLI after microaspiration of milk in rabbits, which was explained by clearance of lipids from alveolar macrophages after aspiration. It is possible that food particles do not reach alveoli during microaspiration and are evacuated from the respiratory system due to ciliary and cough clearance.

As previously stated, the LLI is used as an indicator of aspiration in BALF from humans. It was therefore hypothesized that the quantity of BALF inflammatory cells would correlate with LLI in paediatric patients with chronic respiratory symptoms. However, the data from young children failed to show any correlation between LLI and BALF cellularity. Although it is possible that pulmonary symptoms in children who aspirate are due to noninflammatory events, such as triggering of receptors in large airways, it might be that increased LLI is not completely specific for aspiration in children with chronic respiratory symptoms. This conclusion is consistent with the recent report by KNAUER-FISCHER and RATJEN [19], which suggests that elevated lipid indices can be found in a variety of paediatric pulmonary diseases, independent of aspiration.

It is known that factors other than GER and aspiration can cause accumulation of lipids in alveolar macrophages. For example, alveolar macrophages degrade surfactant and surfactant-like lipids, and byproducts of surfactant metabolism can be detected in their cytoplasm [20]. A significant amount of lipid was found in alveolar macrophages of children with acute chest syndrome due to sickle cell anaemia, and was attributed to pulmonary fat embolism [21]. The lipid indices in this group of patients were significantly higher than in a group of patients with aspiration pneumonia. There are also iatrogenic causes of the accumulation of lipids in alveolar macrophages, such as use of the anti-arrhythmic drug amiodarone, a powerful inhibitor of lysosomal phospholipases [22], and treatment with intravenous fat emulsions [23]. Finally, both mineral oil aspiration [24] and lipidoses with pulmonary involvement [25] can be associated with increased LLI.

A notable finding of the present study was that in children with CF, the LLI was increased compared with both children without $\mathrm{CF}$ and healthy adults, even though the results of their $\mathrm{pH}$ measurement studies were not different from children without CF. This suggests that aspiration was not the most important factor leading to accumulation of increased amounts of lipids in alveolar macrophages in children with CF. One possible explanation for this phenomenon could be that prominent inflammation in the airways of children with CF leads to the accumulation of breakdown products from lipid-containing membranes of inflammatory cells in alveolar macrophages. However, the absence of any correlation of LLI with airway inflammation in CF children in this study makes this explanation less satisfactory. It can also be speculated that children with $\mathrm{CF}$ have prominent airway obstruction and may develop conditions similar to lipoid pneumonia, which was described in patients with lung cancer [26]. It is possible that this process also occurs in children with chronic obstructive pulmonary diseases other than $\mathrm{CF}$.

In summary, it is concluded that the lipid-laden index did not correlate with markers of inflammation in bronchoalveolar lavage fluids in children with chronic respiratory symptoms. Furthermore, the lipidladen index is elevated in bronchoalveolar lavage fluids from children with cystic fibrosis, who do not appear to have greater gastro-oesophageal reflux or clinical aspiration than other children with chronic respiratory symptoms. The lipid-laden index may lack the specificity needed to establish a diagnosis of aspiration in paediatric patients. Further investigation is required to differentiate the multiple potential sources of lipid accumulation in alveolar macrophages.

\footnotetext{
Acknowledgements. The authors thank I. Kazachkova for technical assistance, A. Ghio for assistance with obtaining bronchoalveolar lavage fluids in healthy adults, and the staff of the University of North Carolina Pediatric Bronchology Center for assistance with procedures.
}

\section{References}

1. Euler AR, Byrne WJ, Ament ME, Fonkalsrud EW, Strobel CT, Katz RM. Recurrent pulmonary disease in children: a complication of gastroesophageal reflux. Pediatrics 1979; 63: 47-51.

2. Contencin P, Maurage C, Ployet M, Seid A, Sinaasappel M. Gastroesophageal reflux and ENT disorders in childhood. Ped Otorhinolaryngol 1995; 32: S135-S144.

3. Balson BM, Kravitz EK, McGeady SJ. Diagnosis and treatment of gastroesophageal reflux in children and 
adolescents with severe asthma. Ann Allergy Asthma Immunol 1998; 81: 158-164.

4. Mansfield LE, Stein MR. Gastroesophageal reflux and asthma: a possible reflex mechanism. Ann Allergy 1978; 41: 224-226.

5. Staugas R, Martin AJ, Binns G, Steven IM. The significance of fat-filled macrophages in the diagnosis of aspiration associated with gastro-oesophageal reflux. Aust Paediatr J 1985; 21: 275-277.

6. Nussbaum E, Maggi CJ, Mathis R, Galant SP. Association of lipid-laden alveolar macrophages and gastroesophageal reflux in children. J Pediatr 1987; 110: 190-194.

7. Corwin R, Irwin R. The lipid-laden alveolar macrophage as a marker of aspiration in parenchymal lung disease. Am Rev Respir Dis 1985; 132: 576-581.

8. Colombo JL, Hallberg TK. Recurrent aspiration in children: lipid laden alveolar macrophage quantitation. Pediatr Pulmonol 1987; 3: 86-89.

9. Teabeaut RJ. Aspiration of gastric contents. Am J Pathol 1952; 28: 51-67.

10. Ishii Y, Kobayashi J, Kitamura S. Chemotactic factor generation and cell accumulation in acute lung injury induced by endotracheal acid instillation. Prostaglandins Leukot Essent Fatty Acids 1989; 37: 65-70.

11. Schwartz DJ, Wynne JW, Gibbs CP, Hood CI, Kuck EJ. The pulmonary consequences of aspiration of gastric contents at $\mathrm{pH}$ values greater than 2.5. Am Rev Respir Dis 1980; 121: 119-126.

12. Muhlebach MS, Stewart PW, Leigh MW, Noah TL. Quantitation of inflammatory responses to bacteria in young cystic fibrosis and control patients. Am J Respir Crit Care Med 1999; 160: 186-191.

13. Vandenplas Y, Sacre-Smits L. Continuous 24-hour esophageal monitoring in 285 asympromatic infants 0-15 months old. J Pediatr Gastr Nutr 1987; 6: 220-224.

14. Downs JB, Chapman RL Jr, Modell JH, Hood CI. An evaluation of steroid therapy in aspiration pneumonitis. Anesthesiol 1974; 40: 129-135.

15. Weiser MR, Pechet TT, Williams JP, et al. Experimental murine acid aspiration injury is mediated by neutrophils and the alternative complement pathway. J Appl Physiol 1997; 83: 1090-1095.

16. Kennedy TP, Johnson KJ, Kunkel RG, Ward PA, Knight PR, Finch JS. Acute acid aspiration lung injury in the rat: biphasic pathogenesis. Anesth Analg 1989; 69: 87-92.

17. Irons EE, Apfelbach CW. Aspiration bronchopneumonia with special reference to aspiration of stomach content. JAMA 1940; 115: 584-587.

18. Colombo J, Hallberg T. Airway reactivity following repeated milk aspiration in rabbits. Pediatr Pulmonol 2000; 29: 113-119.

19. Knauer-Fischer S, Ratjen F. Lipid-laden macrophages in bronchoalveolar lavage fluid as a marker for pulmonary aspiration. Pediatr Pulmonol 1999; 27: 419-422.

20. Wright JR, Youmans D. Degradation of surfactant lipids and surfactant protein A by alveolar macrophages in vitro. Am J Physiol 1995; 268: L772-L780.

21. Vichinsky E, Williams R, Das M, et al. Pulmonary fat embolism: a distinct cause of severe chest syndrome in sickle sell anemia. Blood 1994; 83: 3107-3112.

22. Jacobson W, Stewart S, Gresham M, Goddard M. Effect of amiodoron on the lung shown by polarized light microscopy. Arch Pathol Lab Med 1997; 121: 1269-1271.

23. Sherman R, Weiss Y, Liebergall M, Pizov R. Alveolar macrophages fat stain in early diagnosis of fat embolism syndrome. Isr J Med Sci 1997; 33: 654-658.

24. Bandla H, Davis S, Hopkins N. Lipoid pneumonia: a silent complication of mineral oil aspiration. Pediatrics 1999; 103: E19.

25. Tabak L, Yilmazbayhan D, Kilicaslan Z, Tascioglan L, Agan M. Value of bronchoalveolar lavage in lipidoses with pulmonary involvement. Eur Respir $J$ 1994; 7: 409-411.

26. Sulkowska M, Sulkowski S, Chyczewski L, Niklinski J. Surfactant system in lung cancer. Endogenous lipid pneumonia. Neoplasma 1997; 44: 167-171. 